\title{
Aptitud cognitiva y compromiso motivacional en el éxito educativo de estudiantes con y sin dificultades de aprendizaje
}

\section{Cognitive Aptitude and Motivational Commitment in the Educational Success of Students with and without Learning Difficulties}

\author{
Fernando Oliveira Pereira \\ Escola Superior de Educação Almeida Garrett, Lisboa, Portugal \\ Universidad Lusófona Lisboa, Lisboa, Portugal \\ ORCID: https://orcid.org/0000-0003-1040-8926
}

Recibido 20-08-18 Revisado 12-09-18 Aprobado 21-11-18 En línea 19-02-19

Correspondencia

Email: fopereira@sapo.pt

\section{Citar como:}

(c) Universidad San Ignacio de Loyola, Vicerrectorado de Investigación, 2019. 


\section{Resumen}

La estructura de la actividad mental y el consiguiente desarrollo de sus componentes funcionalmente afectan la calidad del desempeño de los estudiantes. Esta investigación tuvo como objetivo conocer la diferencia entre algunos detalles estruturales de los niveles psicológicos de la funcionalidad de los alumnos con y sin dificultades de aprendizaje. Investigación de predominancia cuantitativa y de tipología experimental, teniendo por variables independientes la edad, el año escolar, las retenciones escolares, la aptitud cognitiva y la motivación para aprender y por variables dependientes el rendimiento o éxito escolar. La muestra de esta investigación consistió en un contingente de 550 estudiantes: 275 con y 275 sin dificultades de aprendizaje. Los instrumentos metodológicos usados fueron la entrevista psicológica, cuestionários sociodemográficos, de registro del desempeño de los estudiantes en los tres períodos de los dos últimos años escolares y de evaluación de los maestros y de los padres sobre las habilidades de los alumnos para aprender y la motivación para estudiar. Los resultados muestran que los componentes de la actividad de aprendizaje - aptitud cognitiva, compromiso motivacional y éxito educativo - presentan diferencias importantes en el cuadro de intercorrelaciones entre componentes en los alumnos con dificultades de aprendizaje, siendo menor en estos la consistencia de los vínculos entre los diversos componentes estudiados comparado al cuadro presentado en los alumnos sin dificultades de aprendizage. La discusión demuestra y concluye que la conexión menos fuerte entre los componentes, el rendimiento académico y la aptitud para el aprendizaje y la motivación para estudiar, conduce a la mayor dispersión de los níveles de funcionamiento psíquico de los alumnos con dificultades de aprendizaje.

Palabras clave: Aptitud cognitiva; Motivación; Dificultades de aprendizaje.

\section{Summary}

The structure of mental activity and the consequent development of its components functionally affect the quality of the students' performance. The aim of this research was to know the difference between some structural details of the psychological levels of the functionality of the students with and without learning difficulties. It is a predominantly quantitative and experimental study with independent variables such as age, school year, school retentions, cognitive aptitude and motivation to learn, and with dependent variables such as performance or school success. The sample of this research consisted of 550 students: 275 with and 275 without learning difficulties. The methodological instruments used were psychological interviews, sociodemographic questionnaires, record of the students' performance in the three periods of the last two school years and evaluation by teachers and parents of the students' abilities to learn and motivation to study. The results show that the components of the learning activity - cognitive aptitude, motivational commitment and educational success - present important differences within the framework of intercorrelations between components in the students with learning difficulties in comparison to the students without learning difficulties. In the first case, the consistency of the links between the various studied components is lower. The discussion of the data shows and concludes that the less strong connection between the components of academic performance, aptitude for learning and motivation to study, leads to a greater dispersion of the levels of psychic functioning of students with learning difficulties.

Keywords: Cognitive Aptitude; Motivation; Learning Difficulties.

\section{Introducción}

El alumno como sujeto de la actividad de aprendizaje es un sistema cuya estructura psicológica presenta un grado de desarrollo de sus componentes, lo que puede garantizar el éxito escolar cuando el ingreso es positivo, o no, experimentando entonces dificultades de aprendizaje; la estructura del sistema psicológico comprende cuatro sub-estructuras: emocional, cognitiva, motivacional y volitiva (Pereira, 2008, 2014, 2018). 
Este estudio se centrará en el nivel psicológico y los aspectos estructurales del desarrollo cognitivo y motivacional bajo el ámbito psicoeducativo y psicopedagógico, con el fin de entender cuáles son los factores y componentes que están cubiertos y cuales son los que interfieren en el rendimiento escolar. El objetivo es conocer que caracteriza a los estudiantes con dificultades de aprendizaje, diferenciándolos de aquellos que realmente no revelan dificultades.

\section{Factores estructurales de la actividad escolar en el éxito o fracaso académico.}

Siempre hubo dificultades de aprendizaje, pero los problemas del éxito y del fracaso escolar surgen principalmente de la ampliación del acceso a la educación primaria a toda la población en la edad de asistir a la escolaridad inicial obligatoria (Benavente, 1990).

Por lo tanto, no se puede ignorar la realidad histórica de la institución a la que pertenece el estudiante, al formular juicios sobre el éxito o el fracaso escolar. Esto se debe a que hay diferencias específicas entre lo que se entiende por rendimiento escolar y el éxito educativo. La misma calificación otorgada al estudiante por diferentes instituciones no se corresponde con las mismas habilidades y competencias iguales son evaluadas de manera diferente de institución a institución. Un estudiante promedio puede ser excelente en una clase débil y mediocre en una clase fuerte. Una escuela sólo puede evaluar lo que enseña, no puede confundirse el conocimiento y las habilidades reales de un estudiante con el de la excelencia académica que se evalúa (Perrenoud, 2003). Los estudios han revelado que las causas del fracaso escolar no son las mismas para los padres, estudiantes, maestros, o el sistema educativo. Entre mitos y realidades todavía existen muchas confusiones. Siendo las contradicciones inherentes a la realidad social es probablemente esencial tener en cuenta el contexto histórico, social, político y cultural, en que se inserta el estudiante o la escuela (Roazzi \& Leander, 1988; Nogueira, 1991, 1995; Almeida, Miranda \& Guisande, 2008; Forgiarini \& Silva, 2008; Lira, 2008).

No obstante las diferencias entre las opiniones de los maestros, padres y estudiantes sobre las causas que atribuyen al fracaso escolar el tema se ha centrado, cada vez más, en los problemas de la política educativa y social (Roazzi \& Almeida, 1988). Este enfoque refuerza la posición de Benavente (1990), que la escuela debe considerar las diferencias como diferencias en lugar de deficiencias.

También se sabe que las diferencias individuales de los estudiantes, especialmente en cuanto a sus capacidades de aprendizaje y las motivaciones para estudiar, se puede entender en algunos casos como la riqueza y la diversidad en la forma de alcanzar los objetivos, en los factores que dificultan y obstaculizan el proceso que lleva a éxito académico, sin olvidar mencionar el éxito educacional (Guerra, Candeias \& Prieto, 2014; Lemos, Casanova \& Almeida, 2015; Pinheiro, 2016).

En la literatura no se ha encontrado consenso sobre las dificultades de aprendizaje. Tal vez debido a que estas pueden ser tanto una causa como una consecuencia del fracaso escolar. Las dificultades de aprendizaje tienen causalidad multifactorial (Fonseca, 2004; Correia \& Martins, 2005; Coggi \& Richiardi, 2013) y los factores pueden ser diferentes en estudiantes diferentes. También pueden tener diferentes efectos sobre las actitudes y comportamiento de los estudiantes de acuerdo con las características individuales de la personalidad (Araújo, Lima \& D’Ottaviano, 2013; Alves, 2015; Pässler, Beinicke \& Hell, 2015).

Por lo tanto, las dificultades de aprendizaje deben abordarse en el ámbito de una cadena de causas y consecuencias de los problemas sociales de la edad escolar. El bajo rendimiento académico puede desencadenar sentimientos de baja autoestima, así como también puede influir negativamente en la productividad futura del estudiante, la aceptación por sus compañeros y miembros de la familia, otras actividades relacionadas con el proceso de desarrollo de los niños y otros pasos posteriores (Mazer, Bello \& Bazon, 2009; Sá, 2017). 
El éxito escolar promueve el desarrollo socio-afectivo equilibrado (Elias, 2003; Vianin, 2013). En este sentido, los problemas relacionados con el aprendizaje de los estudiantes pueden ser un factor de riesgo y desencadenar en los niños comportamientos negativos (Sapienzal \& Pedromônio, 2005; Piletti \& Rossato, 2011; Araújo, Lima \& D’Ottaviano, 2013).

Las dificultades de aprendizaje y de comportamiento pueden, simultáneamente, incrementar la probabilidad de la aparición de problemas en contextos sociales y escolares, interferir negativamente en las relaciones interpersonales de los estudiantes, en su adaptación social y también en el concepto de sí mismo (Bianchi, 2005; Sá, 2017).

La mayoría de las investigaciones indican que las dificultades de aprendizaje son factores de riesgo para problemas de carácter psicosocial: baja autoestima, habilidades sociales deficitarias, incluyendo problemas de comportamiento antisocial y de inadaptación social (Mazer, Bello \& Bazon, 2009; Fernandes, 2010; Sá, 2017).

Muchos de estos factores son los componentes de la estructura psíquica del alumno, cada una con sus especificidades. Por lo tanto, se puede hacer referencia a los aspectos estructurales relacionados com las esferas: emocional, cognitiva, motivacional, volitiva (Pereira, 2008, 2014, 2018). Los factores de naturaleza psicológica más comunes en la determinación de los problemas de aprendizaje son el cognitivo y el motivacional (Pissaro, 2012; Barbosa, 2015; Gonçalves, Barreiros, Barreiros \& Correia, 2017).

De ahí la conclusión de que el rendimiento académico de los estudiantes que se caracterizan por el fracaso escolar, no se origina únicamente en los disturbios y las condiciones internas que generan dificultades de aprendizaje individuales. El fracaso escolar y educativo puede tener lugar en los estudiantes con el desarrollo normal de sus condiciones internas encargadas de aprendizaje, siendo una consecuencia de factores, aunque individual, más centrado en el arco de la interacción del sujeto con los diversos contextos del mundo circundante.

\section{Influencia de los componentes - aptitud para aprender y dedicación al estúdio - sobre el rendimiento académico de los alumnos.}

El rendimiento escolar en la condición del éxito y del fracaso escolar y educativo puede verse afectado por factores de la más variada orden y tamaño, siempre que interfieren con el funcionamiento psicológico de los estudiantes en interacción y relación con el mundo.

Hay alumnos cuyo fracaso escolar está relacionado con problemas en el ámbito de las dificultades de aprendizaje, que surgen de las particularidades de sus aptitudes cognitivas o experiencias emocionales en la familia o con los compañeros. Pero también hay estudiantes en que el fracaso escolar depende exclusivamente de su dedicación al estudio y la falta de motivación para las tareas escolares, ya que en otras tareas, por ejemplo ludicas, demuestran un alto rendimiento (Coggi \& Richiardi, 2013; Veríssimo, 2013; Pereira, 2015).

Por lo tanto, los resultados de aprendizaje y en consecuencia el rendimiento académico del estudiante concreto pueden ser explicados por diferentes teorías explícitas: algunos autores recurren a la inteligencia y los procesos cognitivos implicados (Piaget, 1978; Sternberg, 1988; Piletti \& Rossato, 2011), otros a la personalidad y sus componentes, principalmente emocional y afectivo, con la participación de la autoestima (Kelly, 1995; Norenzayan, Choi \& Nisbett, 1999; Sá, 2017), otros consideran que el problema radica en las creencias y estereotipos sociales (Rodrigo, Rodríguez \& Marrero, 1993; Lourenço, 2010). Siendo que otros, con legitimidad teoríca y práctica, dan énfasis al papel de la motivación (Huertas \& Agudo, 2003; Souza, 2010; Vianin, 2013; Gabriel \& Oliveira, 2014), así como aquellos que entienden que la causa del problema se centra en el proceso de socialización y la educación y el aprendizaje (Kember, 1997; Pozo et al, 1999; Máiquez et al, 2000; Pérez Echevarría et al, 2001; Coggi \& Richiardi, 2013; Sá, 2017). 
Brophy (1988) caracteriza la motivación para aprender como la tendencia de que el estudiante debe asignar significado y valor a las actividades académicas. Al parecer, los objetivos, necesidades y creencias de los estudiantes son aspectos que están involucrados directa o indirectamente en la motivación para aprender, y que se dirigirán a esa objetividad que da importancia, significado, valor (Carraça, 2017; Sá, 2017; Tabile \& Jacometo, 2017).

Las causas atribuidas al éxito o fracaso de los estudiantes tienen implicaciones para el enfoque de la motivación, ya que da un relevo a una u otra dimensión (Weiner, 1986, 1994; Nogaro, Ecco \& Rigo, 2014).

La motivación es un factor o proceso psicológico interno, que realiza la función de impulsar y de dirigir la acción, mediante la integración de la conducta del sujeto (Murray, 1986; Garrido, 1990; Piletti \& Rossato, 2011; Leal \& Nogueira, 2012).

En la medida que la motivación es un proceso y no el producto, circunstancialmente tampoco hay acceso a la observación directa; por lo tanto, sólo puede deducirse de la observación y análisis de la conducta, haciendo entonces sentido abarcar otros elementos (Pitrinch \& Schunk, 2002; Siqueira \& Wechsler, 2006; Lourenço \& Paiva, 2010; Gabriel \& Kataoka, 2012). Por lo tanto, en los informes a la motivación para el aprendizaje, se debe tener en cuenta varias características de las actividades escolares, tales como la realización de tareas cuyo éxito depende en cierta medida de los procesos cognitivos implicados: la atención, memória, procesamiento de la información, razonamiento, resolución de problemas (Bzuneck, 2002; Siqueira \& Wechsler, 2006; Miotto, 2012; Mascarello, 2013; Dutra, Ghedin, Ghedin \& Nicot, 2017). Aún más especifica la evidencia de la acción de los procesos cognitivos en las dificultades de aprendizaje es en el ámbito de la lectura y de las matemáticas (Dyson, Jordan \& Glutting, 2011; Willcitt, Petrill, Wu, Boada, Detries, Olson \& Pennington, 2013; Carsol \& Dornelegi, 2015).

La relación de la motivación - aprendizaje es recíproco; donde la motivación tiene efecto sobre el aprendizaje y el aprendizaje también interfiere con la motivación (Schunck, 1991, 1996; Fontaine, 2005; Rosario, 2005; Ventzel \& Wigfield, 2009; Lourenço \& Paiva, 2010; Gabriel \& Oliveira, 2014).

La motivación es un proceso en acción que se caracteriza por componentes inherentes a la individualidad del estudiante: la intensidad que refleja el grado de activación, la dirección de la atención y el esfuerzo enfocado en lo que es relevante para el logro del objetivo y la persistencia que permite la manutención de la acción el tiempo requerido (Lemos, 2005; Veríssimo, 2013).

Sin embargo, el acceso de la motivación a la observación se hace posible con el uso de inferencias resultantes del análisis y la interpretación del comportamiento de los estudiantes. Muy a menudo se toman aspectos de la motivación por aspectos generales de las discapacidades de aprendizaje (Boruchovitch, 2009; Artero, 2012; Araújo, Lima \& D’Ottaviano, 2013). La confusión es más probable que se establezca, porque no hay una teoría unívoca y universal de la motivación, pero si varias teorías de la esfera de las particularidades (Lourenço \& Paiva, 2010; Costa, Silva \& Abbas, 2017). Por lo tanto la motivación del estudiante para el aprendizaje escolar debe ser diseñada en la confluencia de la integración de un conjunto de cogniciones motivacionales. Por un lado, la motivación integra las expectativas de los estudiantes en forma de creencias y anticipaciones relacionadas con la posibilidad de alcanzar los resultados deseados y, por otro lado, el valor que adquieren por sí mismos las actividades, objetivos y resultados (Elliot \& Dweck, 2005; Dweck, 2012). Bajo la motivación en las dificultades de aprendizaje se verifica que a menudo el estudiante revela expectativas y valores negativistas frente a su desempeño; de allí el tener la percepción de baja competencia académica, que a su vez conduce a la anticipación de la probabilidad de fallo, adoptando así una estrategia de evitación de la tarea y de compromiso insuficiente (Guay, Marsh \& Boivin, 2003; Gabriel \& Oliveira, 2014). 
Co-substancialmente, la motivación para el aprendizaje se refleja en los distintos niveles de la funcionalidad mental: de las necesidades - en forma de necesidad de competencia; de los objetivos - en que el desempeño puede ser dirigido por las estrategias de aproximación o de evitación; de las atribucíones y del locus de control - representados como éxito o fracaso; de lo Self - como las expectativas y la auto-eficacia; de la realización - como la conducta motivada o de pobre realización; de las consecuencias - se manifiestan por sentimientos de ánimo o desánimo (Efklides \& Sideridis, 2009; Souza, 2010; Barbosa, 2015).

Como resultado de las diferencias, resultantes de la interpretación de la motivación en un plan de naturaleza reduccionista, la teoría de la autodeterminación surge y concibe la motivación en un continuum ininterrumpido, poniendo en la parte izquierda de un segmento de línea la amotivación o desmotivación; en la parte central tiene la motivación extrínseca, con cuatro modos secuenciales de acuerdo con el grado de implicación hacia la internalización: el externo, el introjectado, el identificado y el integrado; e por ultimo, en la parte derecha del segmento de línea se coloca la motivación intrínseca, que se caracteriza principalmente por el alto grado de autonomía y la competencia del sujeto motivado para realizar la acción con el placer y la satisfacción (Ryan \& Deci, 2000; Deci \& Ryan, 2002; Niemiec, Ryan \& Deci, 2009).

Con base en la reflexión y comprensión de lo expuesto, se decidió investigar en alumnos referenciados por la escuela como teniendo dificultades de aprendizaje, que influencia tienen en el rendimiento escolar del alumno dos de las probables causas del fracaso escolar: la aptitud cognitiva para aprender y la motivación para estudiar.

El objetivo de la investigación conduce a la formulación de algunas cuestiones de orientación de la investigación:

1. Los factores de aptitud cognitiva para aprender, motivacionales y de dedicación al estudio interfieren de igual forma en el rendimiento escolar de alumnos con y sin dificultades de aprendizaje?

2. La dimensión "estructura psicológica del aprendizaje" en la condición de factor con influencia determinante en el rendimiento escolar presenta la misma configuración intercorrelacional de los elementos constituyentes en alumnos con y sin dificultades de aprendizaje?

\section{Método}

\section{Participantes.}

La muestra de esta investigación consistió en un contingente de 550 estudiantes: 275 de ambos sexos, con más hombres que mujeres, referenciados por sus escuelas por presentar dificultades de aprendizaje de los contenidos escolares y 275 estudiantes sanos, que no experimentan ninguna dificultad de aprendizaje y también presentan identidad de las características sociodemográficas. Asímismo esta cuota fue distribuida por grupos idénticos en cuanto a las características correspondientes en el contingente de las dificultades de aprendizaje.

El diagnóstico fue establecido y confirmado en la consulta de psicología del servicio de pediatria, donde son seguidos los alumnos com dificultades de aprendizaje. Para confirmar el diagnóstico de dificultades de aprendizaje se realizó una evaluación psicológica y psicopedagógica orientada a la problemática en cuestión. Los alumnos con y sin dificultades de aprendizaje se distribuyen por diversas edades comprendidas entre los 6 y los 15 años; siendo el promedio de edades - 10,4 años y la desviación estándar - 2,2 años, en los alumnos con dificultades de aprendizaje, y de 10,6 años y 2,3 años respectivamente en los alumnos sin dificultades de aprendizaje. Una parte significativa de estos alumnos - mitad - frecuenta al $1^{\circ}$ ciclo $\left(1^{\circ}, 2^{\circ}, 3^{\circ}\right.$ y $4^{\circ}$ años escolares) y la otra mitad, frecuenta a $\operatorname{los} 2^{\circ}\left(5^{\circ}\right.$ y $6^{\circ}$ años escolares $)$ y $3^{\circ}$ $\left(7^{\circ}, 8^{\circ}\right.$ y $9^{\circ}$ años escolares) ciclos de la enseñanza básica. 
Sin embargo, también se formaron otros dos contingentes constituídos por los padrestutores de los alumnos, cuya finalidad consistió en responder a los cuestionarios y a las entrevistas que formaron parte del proceso de obtención de información sobre la evaluación que hacían de sus hijos como alumnos. Por lo tanto, son 275 padres de alumnos con y 275 sin diagnóstico efectivo de dificultades de aprendizaje.

\section{Diseño de la investigación.}

Investigación de predominancia cuantitativa y de tipología experimental. Después de la identificación de las variables sometidas a la medición no se optó por la metodología de diferenciación del grado de expresión entre las variables, porque ese abordaje ya fue realizado en otro trabajo de investigación (Pereira, 2015). En consecuencia, en esta nueva etapa se consideró pertinente utilizar la metodología de las correlaciones entre pares de variables por separado para los contingentes de alumnos con y sin dificultades de aprendizaje. La finalidad consistió en verificar si había diferencias en los tipos de correlaciones entre variables en los alumnos con dificultades de aprendizaje en comparación con el cuadro que presentaban los alumnos sin dificultades de aprendizaje y también si la cohesión estructural era la misma dentro de los dos contingentes de alumnos.

El procedimiento metodológico de inclusión / exclusión de los alumnos en los contingentes correspondientes a la existencia o ausencia de dificultades de aprendizaje se deriva de la aplicación de varios criterios diferenciadores: la señalización por la escuela basada en la información proporcionada por los docentes del alumno durante los años escolares transcurridos $\mathrm{y}$ actuales, justificando las dificultades del alumno en dominar los contenidos escolares; el escaso rendimiento escolar registrado en los períodos y años escolares respectivos; la autoevaluación hecha por los propios alumnos y la observación de los técnicos de psicología en el ámbito de la evaluación psicológica y psicopedagógica.

A continuación se proporcionó la autorización de los padres-tutores para insertar a sus hijos y ellos mismos en la investigación en curso. Posteriormente los alumnos fueron sometidos al proceso de evaluación y acompañamiento psicológico y psicopedagógico que reforzó y validó el mantenimiento en el contingente de las dificultades de aprendizaje efectivas o en el contingente de la ausencia de dificultades. También los respectivos padres-tutores fueron sometidos a entrevistas dirigidas a las cuestiones en investigación y a responder a los cuestionarios de ámbito sociodemográfico y psicopedagógico sobre las características inherentes a sus hijos relativas a la calificación de las aptitudes cognitivas, motivacionales y de dedicación al estudio. Los datos obtenidos fueron sometidos a análisis estadístico con base en el método correlacional de Pearson y se recurrió al software estadístico SPSS 16.0 (Statistical Program for the Social Sciences).

\section{Instrumentos.}

Entrevista clínico-psicológica de diagnóstico orientado a la confirmación especificada o no, de la existencia de problemas de aprendizaje en los alumnos mencionados e identificados por la escuela como tal, con el propósito de mantener o eliminar estos estudiantes en el contingente al qual pertenecen.

Por medio de la aplicación del método de la entrevista estructurada, los alumnos fueron entrevistados y les fue solicitada hacer autoevaluación de sus aptitudes cognitivas para aprender y de su motivación para estudiar, señalando en una escala cualitativa: malo, mediocre, regular, bueno y excelente, el grado que los caracteriza. Información que permite evaluar cualitativamente el grado de correspondencia de su autoevaluación con la evaluación hecha por los padres-tutores sobre las mismas dimensiones de la investigación. 
Cuestionario sobre las características socio-demográficas y escolares de los estudiantes: de el primer hace parte la identificación de los estudiantes en cuanto a género, edad y situación familiar; del segundo, el año de la asistencia escolar actual, la frecuencia de retención $\mathrm{y}$ en que año escolar tuvo lugar.

Cuestionario del Rendimiento Escolar. Este presenta en forma de tabla o cuadrícula, los registros de las calificaciones obtenidas por el alumno en una escala de valores de 1 a 5 en los tres períodos escolares para el año en curso y del año pasado por asignaturas.

Cuestionario para la medición de competencias, dirigido a los padres-tutores de los estudiantes, cuya estructura consiste en las dimensiones aptitud cognitiva para aprender y compromiso motivacional para aprender y estudiar. Los padres-tutores caracterizan a los estudiantes, en una escala cualitativa, malo, mediocre, regular, bueno o excelente, y posteriormente asignados por el investigador, los valores numéricos - 1, 2, 3, 4, 5 correspondientes a la escala del sistema escolar y relacionados con el rendimiento escolar. Se pide a los padres-tutores de los estudiantes inscribir, en base a su percepción, a que nivel de aptitud cognitiva para el aprendizaje de las asignaturas presenta el alumno, que tan bien conocen. También en el mismo formato se pregunta que grado de motivación para aprender tienen los estudiantes y aún sobre lo que saben acerca de la dedicación de los hijos al estudio.

La confiabilidad de las escalas de los cuestionarios - rendimiento escolar y medición de competencias de los estudiantes asignadas por los padres-tutores - demuestra que el instrumento metodológico cumple los requisitos para los cuales se hizo la medición. Siendo, Cronbach $\alpha=$ 0.71 y 0.91 para las muestras con y sin dificultades de aprendizaje, respectivamente. Los valores obtenidos demuestran existir mayor homogeneidad y cohesión de las respuestas dadas por los padres-tutores a las escalas referentes a las dimensiones constituyentes del cuestionario en el contingente de alumnos sin dificultades de aprendizaje. Por lo tanto, el índice más bajo, aunque con grado de expresión significativo, obtenido en el contingente de los alumnos con dificultades de aprendizaje, muestran la mayor fluctuación de los valores atribuidos por los padres-tutores a las dimensiones cognitivas, motivacionales y de dedicación al estudio de sus hijos, probablemente por éstos también presentan mayor diferenciación en cuanto a las características individuales.

\section{Resultados}

Aqui, en este punto de la estructura que el aprendizaje como fenómeno psicológico global tiene y dónde se quiere entender cómo las variables - el rendimiento escolar, la aptitud cognitiva para el aprendizaje, la motivación para estudiar y de otros factores socio-demográficos, como la edad, años de asistencia a la escuela, fracaso escolar - interaccionan entre sí a fin de producir ciertos efectos en el comportamiento de los alumnos en las escuelas, sólo se muestran las correlaciones que presentan respecto a los niveles de significación estadística superiores a $\mathrm{p}<.05$.

En las figuras 1 y 2, las líneas continuas indican correlaciones positivas, y las líneas discontinuas representan las correlaciones negativas. 
Tabla 1.

Matriz de correlación del rendimiento escolar en los alumnos con dificultades de aprendizaje

\begin{tabular}{|c|c|c|c|c|c|c|c|c|}
\hline VARIABLES & $\frac{\pi}{\tilde{J}}$ & 变 & 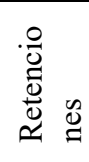 & 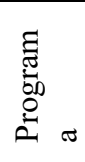 & 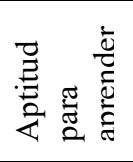 & 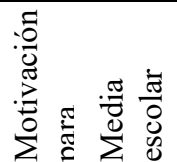 & : & 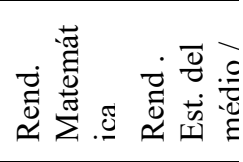 \\
\hline \multicolumn{9}{|l|}{ Edad } \\
\hline \multicolumn{9}{|l|}{ Año escolar } \\
\hline \multicolumn{9}{|l|}{ Retenciones } \\
\hline \multirow{2}{*}{\multicolumn{9}{|c|}{$\begin{array}{l}\text { Programa } \\
\text { adaptado }\end{array}$}} \\
\hline & & & & & & & & \\
\hline $\begin{array}{l}\text { Aptitud para } \\
\text { aprender }\end{array}$ & & & -.232 & & & & & \\
\hline $\begin{array}{l}\text { Motivación } \\
\text { para estudiar }\end{array}$ & -.395 & -.310 & & & .162 & & & \\
\hline $\begin{array}{l}\text { Media Rend. } \\
\text { Escolar }\end{array}$ & -.243 & & & & .421 & & & \\
\hline Rendimiento & & .182 & -.302 & & .361 & .785 & & \\
\hline Portugués & & & & & & & & \\
\hline $\begin{array}{l}\text { Rendimiento } \\
\text { Matemática }\end{array}$ & -.378 & -.235 & -.163 & & .332 & .739 & .328 & \\
\hline Matemática & & & & & & & & \\
\hline $\begin{array}{l}\text { Rend. Est. } \\
\text { médio amb. / } \\
\text { Ciencias }\end{array}$ & -.188 & & & & .277 & .795 & .468 & .404 \\
\hline
\end{tabular}

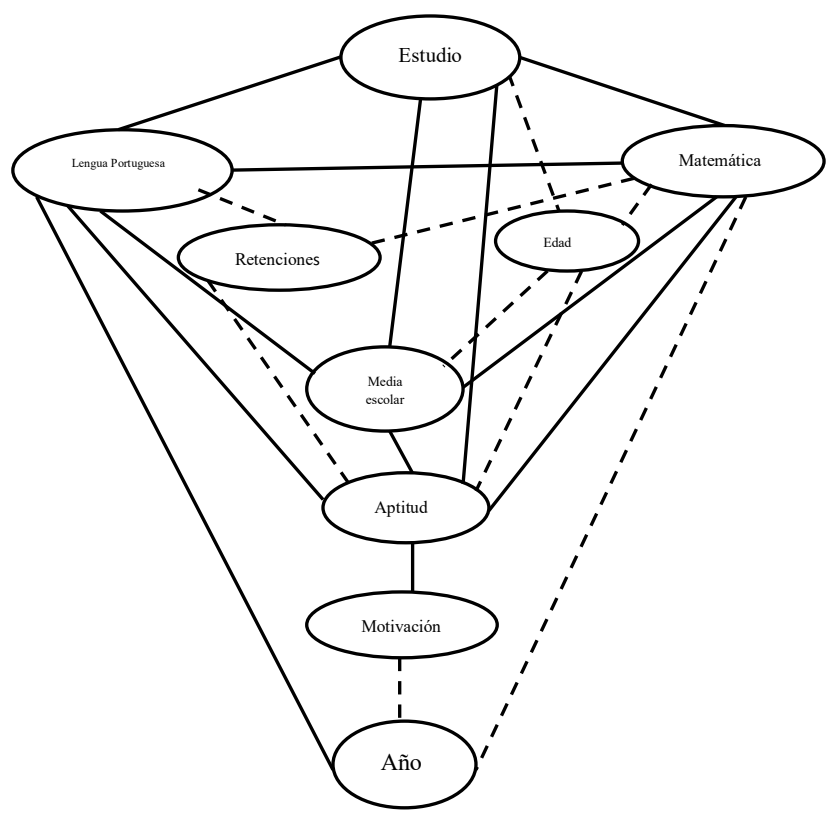

Figura 1. Representación esquemática de la matriz de correlación de rendimiento escolar, aptitudes y motivación en los alumnos con dificultades de aprendizaje

De la estructura funcional formado por las conexiones, representado al mismo tiempo en la tabla 1 y en la figura 1 correspondiente, a nivel psicológico, verifica-se conexiones en los cuales es relativamente débil el índice de influencia en el rendimiento escolar de los alumnos con dificultades de aprendizaje. Los factores socio-demográficos: edad, año escolar y retenciones escolares, se correlacionan negativamente con variables psicológicas - capacidad y motivación y de la escuela en cuanto al rendimiento, que oscila entre los valores de $r=-0.16$ y $r=-0.39$. Luego, a medida que los estudiantes son mayores, están asistiendo a cursos escolares debajo de lo que su edad cronológica, ya que han pasado por las retenciones escolares y con frecuencia 
también ser objeto de programas adaptados a su condición, tiende a revelar niveles más bajos de aptitud para aprender y motivación para estudiar y, en consecuencia, el rendimiento escolar más bajo.

Las características psicológicas - aptitud cognitiva para aprender y motivación para el estúdio - en los estudiantes con dificultades de aprendizaje tienen un índice de interacción pobre $r=0.16$. Siendo que la influencia ejercida por la aptitud en el desempeño escolar en los tres cursos dirigidos varía entre $\mathrm{r}=0.27$ y $\mathrm{r}=0.36$, no existiendo, en este último, cualquier tipo de efecto significativo de la motivación para estudiar. También el rendimiento escolar en los cursos reveló por separado índices de conexión que no parecen ser significativamente más fuertes: $r=0.32$ y $r$ $=0.46$.

Tabla 2

Matriz de correlación del rendimiento escolar en los alumnos sin dificultades de aprendizaje

\begin{tabular}{|c|c|c|c|c|c|c|c|c|c|c|}
\hline Variables & $\underset{\tilde{J}}{\tilde{I}}$ & 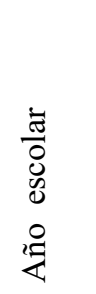 & 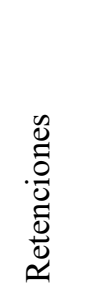 & 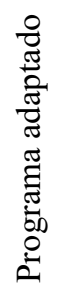 & 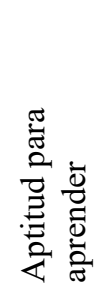 & 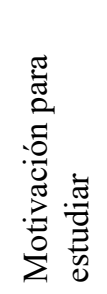 & 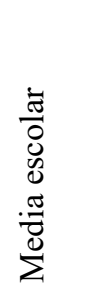 & 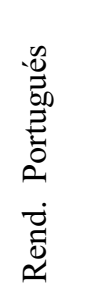 & 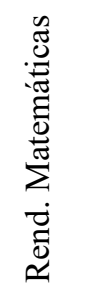 & 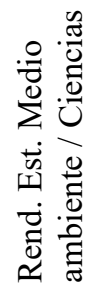 \\
\hline \multicolumn{11}{|l|}{ Edad } \\
\hline Año escolar & .955 & & & & & & & & & \\
\hline Retenciones & .237 & & & & & & & & & \\
\hline \multicolumn{11}{|l|}{$\begin{array}{l}\text { Programa } \\
\text { adaptado }\end{array}$} \\
\hline $\begin{array}{l}\text { Aptitud para } \\
\text { aprender }\end{array}$ & -.491 & -.357 & -.183 & & & & & & & \\
\hline $\begin{array}{l}\text { Motivación para } \\
\text { estudiar }\end{array}$ & -.420 & -.306 & -.424 & & .564 & & & & & \\
\hline $\begin{array}{l}\text { Média Rendim. } \\
\text { Escolar }\end{array}$ & -.633 & -.614 & -.167 & & .636 & .519 & & & & \\
\hline $\begin{array}{l}\text { Rendimiento } \\
\text { Portugués }\end{array}$ & -.561 & -.525 & & & .673 & .514 & .910 & & & \\
\hline $\begin{array}{l}\text { Rendimiento } \\
\text { Matemática }\end{array}$ & -.601 & -.578 & & & .571 & .411 & .942 & .788 & & \\
\hline $\begin{array}{l}\text { Rend. Estudio } \\
\text { medio ambient } \\
\text { /Ciencias }\end{array}$ & -.594 & -.604 & & & .523 & .505 & .927 & .740 & .838 & \\
\hline
\end{tabular}




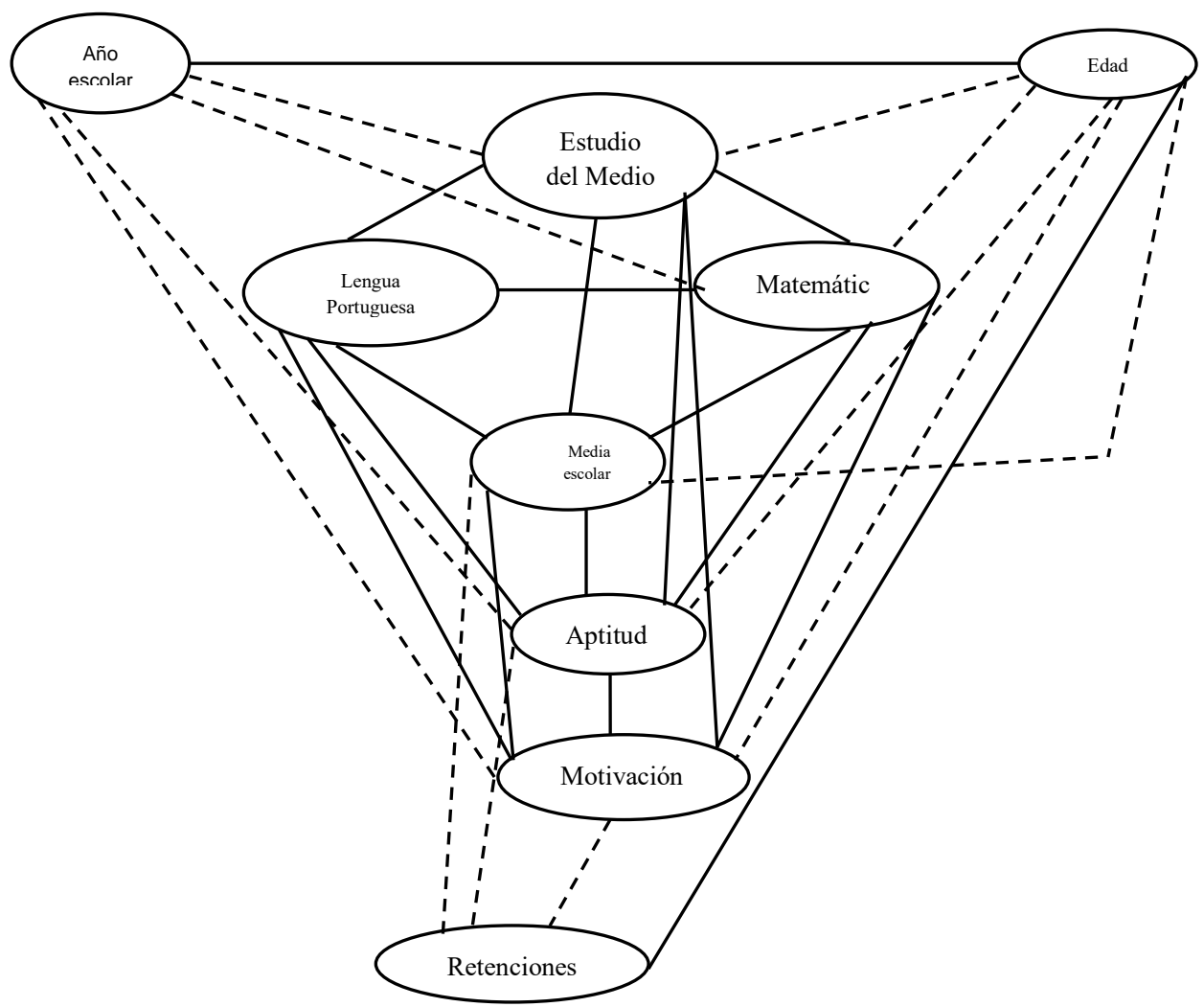

Figura 2. Representación esquemática de la matriz de correlación de rendimiento escolar, aptitud y motivación en los alumnos sin dificultades de aprendizaje

En la estructura funcional formado por las conexiones, representado en la tabla 2 y figura 2 correspondiente, a nivel psicológico, existen conexiones, cuyas tasas de influir en el rendimiento académico de los estudiantes sin discapacidades de aprendizaje son mucho más fuertes, por lo menos en comparación con lo que se encontró en los alumnos con dificultades.

Siguiendo la misma secuencia de la exposición anterior, los factores socio- demográficos: edad, año escolar y retenciones escolares también tienen correlación negativa con las variables psicológicas - capacidad y motivación - y de la escuela en cuanto al rendimiento, pero ahora oscila entre los valores de $r=-0.16$ y $r=-0.60$, predominando valores cercanos al umbral superior. Del mismo modo, como el aumento de los valores relacionados con variables sociodemográficas bajarán las tasas de aptitud para aprender y motivación para estudiar $\mathrm{y}$, en consecuencia, el rendimiento escolar más bajo. Sin embargo, estos estudiantes no experimentan dificultades de aprendizaje y por qué su rendimiento académico es positivo. De ahí la diferencia en la cuota consiste en el hecho de que estos estudiantes generalmente asisten a la escuela en el año de acuerdo con su edad cronológica, no fueron objeto de retención o programas de adaptación, por lo menos tan dependiente de su capacidad de aprender. Puede ser que en algunos casos debido a factores motivacionales del desinterés y la falta de dedicación al estudio algunos de los estudiantes de esta cuota podría haber sido objeto de algunas medidas pedagógicas orientadas al restablecimiento de su funcionamiento.

En cuanto a las características psicológicas - aptitud cognitiva para aprender y motivación para estudiar - en este contingente de estudiantes sin dificultades de aprendizaje el índice de interacción es alta $r=0.56$. La variación de la influencia ejercida por los factores psicológicos en el rendimiento escolar en los tres cursos dirigidos, $r=0.41-0.67$. Sin embargo, el rendimiento escolar obtenido por separado para cada uno de los cursos expresó índices de conexión significativamente más altos: $\mathrm{r}=0.74-0.83$. 


\section{Discusión}

En cuanto a la caracterización socio- demográfica y condición de asistencia escolar de los alumnos con dificultades de aprendizaje se demostró que en este contingente, objeto de la investigación, la tendencia es que estos estudiantes presentan edad cronológica más avanzada, años de escolaridad más bajos, más retenciones escolares y adaptaciones curriculares respecto a lo que ocurre en los estudiantes que no están incluídos, por razones obvias, en la categoría de dificultades de aprendizaje.

El rendimiento académico del contingente de estudiantes con dificultades de aprendizaje también es demostrativo de tales dificultades; es absolutamente visible en la tendencia principal de quedarse en el rango representante de evaluaciones negativas, tanto en la media general del total de evaluaciones, tales como los resultados obtenidos en cada unidad curricular en separado.

Sin embargo, los componentes relacionados con la estructura psicológica de los estudiantes, teniendo en cuenta las evaluaciones que se hicieron de ellos, no es tan perentoria la demostración de la existencia, en este contingente de estudiantes, de las dificultades reales de aprendizaje. Si bien es claro que la mayoría de los estudiantes constituyentes del contingente connotado con dificultades de aprendizaje tienen déficits significativos en la capacidad o aptitud para aprender, hay algunos estudiantes que no tienen precisamente una falta de capacidad cognitiva para dominar el currículo escolar, pero tales como los alumnos anteriores alcanza valores negativos en el rendimiento escolar. En estes estudiantes el fracaso escolar probablemente es más cuestión de falta de motivación para estudiar, traducido por déficit de dedicación al proceso de estudio. Sin embargo, los valores resultantes de la evaluación à la operacionalización activa de este componente motivacional tiende a sugerir que todos, o casi todos, los alumnos del contingente de las dificultades de aprendizaje denotan índices motivacionales débiles e interés en el proceso de estudio, que se manifiesta sobre todo en la débil dedicación al estudio de las materias escolares.

Consecuentemente, los estudiantes con dificultades de aprendizaje tienen tasas más altas en el componente socio-demográfico representado por la retención del año escolar, la frecuencia de ciclo escolar y la edad cronológica; tasa por debajo del componente educativo-pedagógico, relacionada al rendimiento escolar en los diferentes cursos, y en el componente psicológica con respecto a la aptitud cognitiva para aprender y motivación para el estudio, en comparación con los estudiantes que no tienen dificultades de aprendizaje (Artero, 2012; Leal \& Nogueira, 2012; Veríssimo, 2013).

La estructura psicológico del aprendizaje y del consecuente rendimiento académico denuncian diferencia de especificidad entre los dos contingentes de estudiantes: con y sin dificultades de aprendizaje. En el primer contingente, la estructura del rendimiento escolar alcanzado en varios cursos en separado presenta conexiones de sustentación, las cuales, apesar de significativas, son reveladoras de la debilidad de la cohesión y la coherencia entre los valores obtenidos. Refuerza y confirma esta posicion la débil o mediana conexion del rendimento escolar alcanzado a la capacidad o aptitud para aprender y dominar los contenidos impartidos en la escuela, aunque es evidente que los resultados escolares depende en gran medida de las habilidades cognitivas de los estudiantes. Al parecer, la estructura sugiere que otros factores interfieren con el rendimiento académico. Por lo tanto la motivación para estudiar presenta un solo enlace muy débil y solo a la capacidad de aprender, no existiendo conexiones que demuestran la influencia positiva directa en el rendimiento de los estudiantes. La falta de interés de los alumnos por las tareas escolares de aprendizaje potencia más aún la descompensación debido a la falta de capacidades que faciliten el proceso.

Este contingente de estudiantes con dificultades de aprendizaje debido al bajo nivel de desarrollo de las funciones cognitivas responsables de la operación de razonamiento se ve reforzada negativamente por la falta de motivación para el estúdio, consagrado en el completo 
desinterés de los estudiantes mediante la realización de tareas y acciones que sólo pueden mejorar sus actuaciones. Parece que una parte componente del contingente de estudiantes anteriormente referido probablemente tienen habilidades para aprender contenidos escolares, lo que le permite lograr un rendimiento escolar exitoso, aunque mediano, si es que eran titulares de motivación para el estúdio; si fue posible llegar a ser interesados y participar en la adquisición y el dominio de los conocimientos relacionados con el currículo escolar. Obviamente, la implicación aquí conseguir más éxito en la escuela y no tanto el éxito educativo, como se ha informado por algunos autores (Arends, 1995; Perrenoud, 2003; Pozo, 2005; Leal \& Nogueira, 2012; Veríssimo, 2013).

Interpretación reforzada y algo confirmado por la estructura de aprendizaje y el rendimiento escolar existente en el contingente de alunos sin dificultades de aprendizaje. En este caso, los enlaces que apoyan la consistencia e incluso la infalibilidad entre el rendimiento académico alcanzado en los diferentes cursos son muy fuertes. Del mismo modo, la fuerza imprimida por la capacidad de aprender es notable y aclara el papel desempeñado por estas en la aprendizaje y en consecuencia en el rendimiento escolar. Además, la motivación para el estudio, en este contingente de estudiantes, tiene fuertes vínculos con la capacidad de aprender y el rendimiento académico en todas las unidades. Esto demuestra que para tener éxito en la escuela, especialmente el nivel de suceso educativo, es indispensable las funciones positivamente realizadas en la estructura psicológica, ya sea por la aptitud cognitiva para el aprendizaje, ya sea por la motivación para estudiar. Confirmación emergente que algunos estudiantes han revelado suficiente capacidad cognitiva para aprender, pero presentan motivación para estudiar deficitário, observado con facilidad en el desinterés que estos mismos estudiantes tienen en el proceso de estudio, que se refleja en el rendimiento escolar inestable (Artero, 2012; Nogaro, Ecco \& Rigo, 2014). El rendimiento escolar positivo no depende exclusivamente de la capacidad cognitiva para aprender, no siendo raras las situaciones de rendimiento escolar negativo determinado principalmente por el bajo grado de motivación para estudiar (Tabile \& Jacometo, 2017). Entonces, se puede concluir que el rendimiento escolar negativo en los alumnos con dificultades de aprendizaje sobre todo tiene el sello del insuficiente desarrollo de la capacidad de aprender, y el bajo nivel de motivación para el estudio refuerza el agravamiento negativo del rendimiento académico. En particular, en este contingente hay estudiantes que tienen débiles habilidades para aprender, pero lo nivele razonable de motivación para el estudio y la dedicación al proceso de estudio puede lograr resultados satisfactorios en el rendimiento escolar, aunque tolerables. Por el contrario, en los alumnos sin dificultades de aprendizaje el rendimiento escolar débil y negativo es causado principalmente por el muy bajo índice de motivación y dedicación al estudio y nunca, o casi nunca, por falta de capacidad para aprender (Barbosa, 2015; Dutra, Ghedin, Ghedin \& Nicot, 2017).

Por consiguiente, la estructura psicológica, psico-educativa, psicopedagógica del aprendizaje, como una actividad, en estudiantes diagnosticados con dificultades de aprendizaje presentan niveles débiles de la consistencia y homogeneidad de correlación entre los componentes: el rendimiento académico, la aptitud para aprender y motivación para el estudio, lo que demuestra la alta variabilidad y especificidad individuales de los problemas experimentados por estos estudiantes. Los déficit de los recursos cognitivos de los alumnos y la consiguiente conciencia de su falta de capacidad para aprender genera en ellos actitudes de evitación del fracaso en la realización de tareas con los contenidos escolares, lo que refleja negativamente en la motivación y la dedicación al proceso de estudio, cuyos niveles bajos agravan las dificultades de aprendizaje. Explicación que puede deducirse por inferencia de la comparación, en términos interpretativos, con la estructura presentada por los alumnos sin dificultades de aprendizaje; en que el índice de fiabilidad de la cohesión, la consistencia y la relación de homogeneidad entre los componentes son altos y muy significativos. 


\section{Referencias}

Almeida, L. S., Miranda, L., \& Guisande, M. A. (2008). Atribuições causais para o sucesso e fracasso escolares. Estudos de Psicologia. Campinas, 25(2), 169 - 176. Doi: https://doi.org/10.1590/S0103-166X2008000200001 .

Alves, A. F. (2015). Inteligência e rendimento escolar na infầncia: implicações para a sala de aula. Revista de Estúdios y Investigación en Psicología y Educación, 2(2), 113 - 121. Doi: https://doi.org/10.17979/reipe.2015.2.2.1329

Araújo, T. F., Lima, T. O., \& D’Ottaviano, F. G. (2013). Transtornos de aprendizagem na infância: uma revisão da literatura. Revista de Pediatria Moderna, 49(4), 149 - 155. Recuperado de: http://www.moreirajr.com.br/revistas.asp?fase=r003\&id_materia=5371

Arends, R. I. (1995). Aprender a ensinar. Lisboa: McGraw-Hill.

Artero, T. T. (2012). A motivação e sua relação com os problemas de aprendizagem. Eletrônica, Gestão \& Saúde, 3(3). 122 - 132. Doi: https://doi.org/10.18673/gs.v3i3.23064

Barbosa, P. de S. (2015). Dificuldades de aprendizagem. São Luís: UemaNet.

Benavente, A. (1990). Insucesso escolar no contexto português - abordagens, concepções e políticas. Análise Social, 25(108), 715-733. Recuperado de: https://www.jstor.org/stable/41010838

Bianchi, S. H. (2005). Eventos de vida, autoeficácia e autoconceito de crianças com bom desempenho escolar e dificuldades comportamentais (Tesis doctoral). Universidade de São Paulo, Ribeirão Preto, SP.

Boruchovitch, E. (2009). A motivação do aluno (4. ${ }^{\mathrm{a}}$ ed.). Rio de Janeiro: Editora Vozes.

Brophy, J. (1988). On motivating students. In D. Berliner \& B. Rosenshine (Eds.), Talks to teachers (pp. 201 - 245). New York: Random House.

Bzuneck, J.A. (2002). A motivação do aluno: aspectos introdutórios. In Boruchovitch, E. e Bzuneck, J.A. (Orgs.). Motivação do aluno (pp. 9-36). Petrópolis: Vozes.

Carraça, E. V. (2017). Um modelo motivacional de envolvimento dos jovens nas aulas de educação física. Retos, 31, 282-291. Recuperado de: https://recyt.fecyt.es/index.php/retos/article/view/53504

Coggi, C., \& Richiardi, P. (2013). Dificuldades de aprendizagem e insucesso escolar: uma cooperação internacional entre Itália e Brasil. Debates em Educação, 5(9), 14-38. Doi: https://doi.org/10.28998/2175-6600.2013v5n9p14

Correia, L. M., \& Martins, A. P. (2005). Dificuldades de Aprendizagem. O que são? Como entendê-las? Biblioteca Digital. Coleção Educação. Portugal, Porto Editora.

Corsol, L. V., \& Dornelesi, B. V. (2015). Perfil cognitive dos alunos com dificuldades de aprendizagem na leitura e matemática. Psicologia: Teoria e Prática, 17(2). 185-198. Recyuperado de: https://www.redalyc.org/html/1938/193841504014/

Costa, M. A. S., Silva, B. N., \& Abbas, K. (2017). Motivações e estratégias de aprendizagem dos discentes em contabilidade de custos e a influência no desempenho academico. XXIV Congresso Brasileiro de Custos. Florianopólis, SC. Brasil, 15 a 17 de Novembro de 2017.

Deci, E.L., \& Ryan, R.M. (2002). Handbook of self-determination research. Rochester: The University of Rochester Press.

Dutra, A. C., Ghedin, I., Ghedin, L., \& Nicot, Y. (2017). Processos cognitivos: considerações acerca das dificuldades de aprendizagem. Revista Areté / Revista Amazônica de Ensino de Ciências, 6(10), 73-86. Recuperado de: http://periodicos.uea.edu.br/index.php/arete/article/view/62

Dyson, N. Jordan, N., \& Glutting, J. (2011). A number sense intervention for low income kindergartners at risk for mathematics difficulties. Journal of Learning Disabilities, Chicago, 42(2), 166 - 181. Doi: https://doi.org/10.1177/0022219411410233

Dweck, C. S. (2012). Mindset: How You Can Fulfill Your Potential. New York: Constable \& Robinson Limited.

Efklides, A. \& Sideridis, G. D. (2009). Assessing Cognitive Failures. European Journal of Psychological Assessment, 25, 69 - 72. Doi: https://doi.org/10.1027/1015-5759.25.2.69 
Elias, L. C. S. (2003). Crianças que apresentam baixo rendimento escolar e problemas de comportamento associados: caracterização e intervenção (Tesis doctoral). Universidade de São Paulo, Ribeirão Preto, SP.

Elliot, A. J., \& Dweck, C. S. (2005). Handbook of competence and motivation. New York: Guilford.

Fernandes, A. M. (2010). Alfabetização e letramento: definições de conceitos, apresentação de alguns dados sobre o fracasso escolar e discussão do papel social da escola. UFSCar: Revista. São Carlos: Linguagem. Recuperado de: http://www.letras.ufscar.br/linguasagem/edicao13/ref 01.pdf

Fonseca, V. (2004). Dificuldades de aprendizagem. Lisboa: Âncora Editora.

Fontaine, A. M. (2005). Motivação em contexto escolar. Lisboa: Universidade Aberta.

Forgiarini, S. A. B., \& Silva, J. C. da (2008). Fracasso escolar no contexto de escola pública: entre mitos $e$ realidades. Recuperado de: http://www.diaadiaeducacao.pr.gov.br/portals/pde/arquivos/369-4.pdf

Gabriel, F. F., \& Oliveira, M. H. P. (2014). Factores motivacionais na autoregulação da aprendizagem de estatística de estudantes de psicologia. Recuperado de: http://funes.uniandes.edu.co/5339/1/GaribelFatoresALME2014.pdf

Gabriel, F. F. \& Kataoka, V. Y. (2012). A interação professor - aluno na construção de estratégias de autorregulação da aprendizagem estatística. Anais da XXV Reunión Latinoamericana de Matemática Educativa. Brasil: Pontífica Universidade Católica de Minas Gerais PUC/Minas. Recuperado de: http://funes.uniandes.edu.co/4416/1/PalmaAintera\%C3\%A7\%C3\%A2oALME2013.pdf

Garrido, I. (1990). Motivacion, emocion y accion educativa. In Mayor, L. \& Tortosa, F. (Ed) Âmbitos de aplicacion de la psicologia motivacional (pp. 284-343). Bilbao: Desclee de Brower.

Gonçalves, G. dos S., Barreiros, M. O., Barreiros, Sh. P. O., \& Correia, L. C. (2017). Análise dos factores que causam dificuldades de aprendizagem de leitura e escrita nas series iniciais do ensino fundamental. Revista Espacios, 38(60), 01-11. Recuperado de: https://www.revistaespacios.com/a17v38n60/a17v38n60p11.pdf

Guay, F., Marsh, H. M., \& Boivin, M. (2003). Academic Self-concept and academic achievement: Developmental perspectives and their causal ordering. Journal of Educational Psychology, 95(1), 124-136. 124 - 136. Doi: http://dx.doi.org/10.1037/0022-0663.95.1.124

Guerra, C. G., Candeias, A. \& Prieto, G. (2014). Flexibilidade cognitiva: repensar o conceito e a medida da inteligência. In L. S. Almeida, A. M. Araújo, A. R. Franco \& D. L. Soares (2014). Cognição, aprendizagem e rendimento. Braga: Centro de Investigação em Educação.

Huertas, J. A., \& Agudo, R. (2003). Concepciones de los estudantes universitários sobre la motivación. In C. Monereo \& J. I. Pozo (Eds.). La universidade ante la nueva cultura educativa: enseñar y aprender para la autonomia. Madrid: Síntesis.

Kelly, G. (1995). A theory of personality: the psychology of personal constructs. New York: Norton.

Kember, D. (1997). A reconceptualization of the research into university academic conceptions of learning. Learning and Instruction, 7(3), $225-275 . \quad$ Doi: https://doi.org/10.1016/S0959-4752(96)00028-X

Leal, D., \& Nogueira, M. O. G. (2012). Dificuldades de aprendizagem: um olhar psicopedagógico. Curitiba: Intersaberes.

Lemos, M. S. (2005). Motivação. In G. Miranda \& S. Bahia (Orgs.). Psicologia da Educação: Temas de desenvolvimento, aprendizagem e ensino (pp. 193 - 231). Lisboa: Relógio de Água Editores.

Lemos, G. C., Casanova, J. R. \& Almeida, L. S. (2015). Habilidades cognitivas e interesses vocacionais na adolescência: promovendo percursos de sucesso. In G. C. Lemos \& L. S. Almeida (Ed.s). Cognição e Aprendizagem: promoção do sucesso escolar. Braga: Associação para o Desenvolvimento á Investigação em Psicologia da Educação. 
Lira, G. D. (2008). Fracasso escolar: visão de professores das séries iniciais do ensino fundamental da cidade de Cajazeiras PB. Tese. Lisboa: Universidade Lusófona de Humanidades e Tecnologias.

Lourenço, A. A., \& Paiva, M. O. A. de (2010). A motivação escolar e o processo de aprendizagem. Ciências \& Cognição, 15(2),132-141. Recuperado de: http://cienciasecognicao.org/revista/index.php/cec/article/view/313

Máiquez, M. L., Rodrigo, M. J., Capote, C., \& Vermaes, I. (2000). Aprender en la vida cotidiana. Um programa experiencial. Madrid: Visor.

Mascarello, L. J. (2013). As características cognitivas e comportamentais de crianças com baixa memória de trabalho. Linguagem e Ensino, 16(2), 463-480. Recuperado de: http://revistas.ucpel.tche.br/index.php/rle/article/view/892

Mazer, Sh. M., Bello, A. C. D., \& Bazon, M. R. (2009). Dificuldades de Aprendizagem: revisão de literatura sobre os fatores de risco associados. Psicologia da Educação, 28(1)7-21. Recuperado de: http://pepsic.bvsalud.org/pdf/psie/n28/v28a02.pdf

Miotto, E. C. (2012). Avaliação neuropsicológica e funções cognitivas. In Miotto, E. C., Lucia, M. C. S. \& Scaff, M. Neuropsicologia Clínica. Rio de Janeiro: Roca.

Murray, E.J. (1986). Motivação e emoção. Rio de Janeiro: Guanabara-Koogan.

Nogaro, A., Ecco, I., \& Rigo, L. F. R. (2014). Aprendizagem e factores motivacionais relacionados. Espaço Pedagógico, 21(2), 419-434. Doi: https://doi.org/10.5335/rep.v21i2.4309

Nogueira, M. A. (1991). Trajectórias escolares, estratégias culturais e classes sociais. Teoria e Educação, $\mathrm{n}^{\circ}$ 3. Porto Alegre.

Niemiec, Ch. P., Ryan, R. M., \& Deci, E. L. (2009). The path taken: consequences of attainine intrinsec and extrinsic aspirations. Journal of Research in Personality, 43(3), 291 - 306. Doi: https://doi.org/10.1016/j.jrp.2008.09.001

Nogueira, M. A. (1995). Convertidos e Oblatos - Um exame da relação de Classes médias/Escolas na obra de Pierre Bourdieu. Mimeo.

Norenzayan, A., Choi, I., \& Nisbett, R. E. (1999). Eastern and western perceptions of causality for social behavior: lay theories about personalities and social situations. In D. Prentice $\&$ D. Miller (Eds.). Cultural divides: understanding and overcoming group conflict. New York: Sage.

Pässler, K., Beinicke, A., \& Hell, B. (2015). Interests and intelligence: A meta-analysis. Intelligence, 50, 30 - 40. Doi: https://doi.org/10.1016/j.intell.2015.02.001

Pereira, F. O. (2008). Especificidade psicológica da imagem representacional dos estilos personalístico-comportamentais dos profissionais de educação. Porto: Edições Ecopy.

Pereira, F. O. (2014). Educação e Neuroses. Lisboa: Sinapis Editores.

Pereira, F. O. (2015). Especificidades do rendimento, aptidão e motivação escolares em alunos com dificuldades de aprendizagem. Revista Quadrimestral da Associação Basileira de Psicologia Escolar e Educacional, 19(3), 525-536. Recuperado de: http://www.scielo.br/pdf/pee/v19n3/2175-3539-pee-19-03-00525.pdf

Pereira, F. O. (2018). Teoria sistémico-integrativa do psiquismo humano. Teoría y Critica de la Psicología, 10, 1-23. Recuperado de: http://www.teocripsi.com/ojs/index.php/TCP/article/view/209

Pérez Echevarría, M. P., Mateos, M. M., Pozo, J. I., \& Scheuer, N. (2001). En busca del constructivismo perdido: concepciones implícitas sobre el aprendizagem. Estudios de Psicología, 22(2), 155 - 173. Doi: https://doi.org/10.1174/021093901609479

Perrenoud, Ph. (2003). Sucesso na escola: só o currículo, nada mais que o currículo! Cadernos de Pesquisa, 119, 7-26. Recuperado de: http://www.scielo.br/pdf/\%0D/cp/n119/n119a01.pdf

Piaget, J. (1978). O nascimento da inteligência da criança, $3^{\mathrm{a}}$ ed. Rio de Janeiro: Zahar Editores.

Piletti, N. \& Rossato, S. M. (2011). Psicologia da aprendizagem: da teoria do condicioamento ao construtivismo. São Paulo: Contexto.

Pinheiro, R. P. Q. (2016). Aprende-te: O papel do não cognitivo na aprendizagem (Tesis de maestría). ISPA, Lisboa. 
Pissaro, S. P. F. M. (2012). Variáveis cognitivas, cognitivas-motivacionais e rendimento escolar em alunos com dificuldades de aprendizagem (Tesis de maestría). Universidade Lusíada do Porto, Porto

Pitrinch, P. R. e Schunk, D. H. (2002). Motivation in education - theory, research and applications. New Jersey: Merril Prentice Hall.

Pozo, J. I., Scheuer, N., Pérez Echevarría, M. P. \& Mateos, M. (1999). El cambio de las concepciones de los professores sobre el aprendizagem. In J. M. Sanchez, A. Onorbe \& I. Bustamante (Eds.). Educación Científica. Madrid: Ediciones de la Universidad de Alcalá.

Pozo, J. I. (2005). Aquisição de conhecimento. Porto Alegre: Artmed.

Rodrigo, M. J., Rodriguez, A. \& Marrero, J. (Eds.) (1993). Las teorias implícitas: una aproximación al conocimiento cotidiano. Madrid: Visor.

Roazzi, A. \& Almeida, L. S. (1988). Insucesso escolar: insucesso do aluno ou insucesso do sistema escolar? Revista Portuguesa de Educação, 1(2), 53 - 60.

Rosário, P. (2005). Motivação escolar. Uma rota de leitura. In M. C. Taveira (Coord.). Psicologia Escolar: uma proposta científico-pedagógica (pp. 23 - 60). Coimbra: Quarteto.

Ryan, R. M., \& Deci, E. L. (2000). Self-determination theory and the facilitation of intrinsic motivation, social development, and well-being. Am. Psychologist, 55(1), 68-78. Recuperado de: http://www.ppfa.pt/wp-content/uploads/2017/04/Deci-Ryan-2000.pdf

Sá, A. M. S. (2017). Motivação e aprendizagem: A influência familiar na vida escolar dos alunos. InterEspaço: Revista de Geografia e Interdisciplinaridade, 3(11), 149 - 167. Doi: http://dx.doi.org/10.18764/2446-6549.v3n11p149-167

Sapienzal, G., \& Pedromonico, M. R. M. (2005). Risco, proteção e resiliência no desenvolvimento da criança e do adolescente. Psicologia em Estudo, 10(2), pp. 209-216.

Schunk, D. H. (1991). Self-efficacy and academic motivation. Educational Psychologist, 26, $207-$ 231. Doi: https://doi.org/10.1080/00461520.1991.9653133

Schunk, D. H. (1996). Goal and self-evaluative influences during childrens' cognitive skill learning. American Educational Research Journal, 33, 359-382. Doi: https://doi.org/10.3102/00028312033002359

Siqueira, L. G. G., \& Wechsler, S. M. (2006). Motivação para a aprendizagem escolar: possibilidade e medida. Avaliação Psicológica, 5(1), 21-31. Recuperado de: http://pepsic.bvsalud.org/pdf/avp/v5n1/v5n1a04.pdf

Souza, L. F. N. I. (2010). Estratégias de aprendizagem e factores motivacionais relacionados. Educar, 36, 95 - 107. Doi: http://dx.doi.org/10.1590/S0104-40602010000100008

Sternberg, R. J. (1988). Inteligencia humana (4 vol.). Barcelona: Paidós.

Tabile, A. F., \& Jacometo, M. C. D. (2017). Factores influenciadores no processo de aprendizagem: um estudo de caso. Revista de Psicopedagogia, 34(103), 75 - 86. Recuperado de: http://pepsic.bvsalud.org/pdf/psicoped/v34n103/08.pdf

Veríssimo, L. (2013). Motivar os alunos, motivar os professors: Faces de uma mesma moeda. In Machado, J. \& Alves, J. M. (Org.). Melhorar a escola: sucesso escolar, disciplina, motivação, direção de escolas e politicas educativas. Porto: Universidade Católica Portuguesa.

Vianin, P. (2013). Estratégias de apoio a alunos com dificuldades de aprendizagem. São Paulo: Editora Penso.

Weiner, B. (1986). Attribucional theory of motivation and emotion. New York: Springer.

Weiner, B. (1994). Ability versus effort revisited: The moral determinants of achievement evaluation an achievement as a moral system. Educational Psychologist, 29, 163 - 172. Doi: https://doi.org/10.1207/s15326985ep2903_5

Wentzel, K. \& Wigfield, A. (2009). Handbook of Motivation at School. New York: Routledge.

Willcutt, E. G., Petrill, S. A., Wu, S., Boada, R., Detries, J. C., Olson, R. K. \& Pennigton, B. F. (2013). Comorbidity between reading disability: concurrent psychopathology, functional impairment and neuropsychological functioning. Journal of Learning Disabilities, 46(6), 500 - 516. Doi: https://doi.org/10.1177/0022219413477476 